\title{
La guerra del pisco a través del estudio de los marbetes
}

\author{
The war of pisco through the study of the labels \\ Carolina Cofré ${ }^{1}$,Emiliano Núñez ${ }^{1}$, Fernando Mujica ${ }^{2}$ y Pablo Lacoste ${ }^{3 *}$
}

\section{RESUMEN}

Este artículo examina la evolución del Pisco (aguardiente de uva con Denominación de Origen) chileno a partir de las etiquetas registradas en el INAPI desde el inicio (1882) hasta la fecha a delimitación del Pisco como DO (1931). En este periodo se detectaron 111 etiquetas de Pisco. Se compara con las etiquetas de pisco peruano de esa época. También se examina la construcción del nombre del producto (pisco), el origen geográfico (Norte Chico de Chile) y variedades de uva (Moscatel de Alejandría y Moscatel Rosada). Se demuestra que el Pisco chileno definió su identidad 40 años antes que el peruano, lo que explica por qué fue delimitado por ley 60 años antes.

Palabras clave: pisco chileno, pisco peruano, aguardientes, industria vitivinícola, Denominación de Origen.

\begin{abstract}
This paper examines the evolution of Chilean Pisco (kind of grape brandy with Appellation of Origin (AO)), from the labels of brands registered in the National Industrial Property Institute (INAPI, in Spanish) from the first one in 1882, until near the date of the delimitation of Pisco as an AO (1931). During this period, 111 labels of Pisco were detected. The article compares these designs with Peruvian labels from the same time. It also examines the construction of the name of the product (Pisco), the geographic origin (Norte Chico of Chile) and grape varieties (Muscat of Alexandria and Moscatel Rosada). Here, it is demonstrated that Chilean Pisco defined its identity 40 years before the Peruvian, which explains why received its denomination by law 60 years earlier.

Key words: chilean pisco, peruvian pisco, brandy, wine industry, Appellation d'Origine.
\end{abstract}

\section{Introducción}

El Pisco es la primera Denominación de Origen de América y a la vez la única DO reivindicada por dos países, Chile y Perú (Gutiérrez, 2005; Huertas, 2012; Dargent Chamot, 2013; Jiménez, 2014; Lacoste, 2013, 2014). Se trata de un caso singular en el desarrollo de los productos agroalimentarios con identificación geográfica en la región. Porque, a diferencia de Europa, donde existe una sólida cultura de la apreciación de los productos típicos y las DO, en América del Sur este proceso apenas está en vías de desarrollo. En los últimos años se han realizado algunos esfuerzos para la consolidación de productos en Brasil (Souza, 2014), México (Hernández, 2013; López, 2015), Chile (Belmar, 2015, Castro, 2015; Arancibia, 2015) y Bolivia (Aillón, 2013; Buitrago, 2015). De todos modos, se trata de esfuerzos incipientes; hasta ahora ningún producto típico sudamericano ha alcanzado el nivel de desarrollo y relevancia económica, social y cultural del Pisco. Por este motivo, resulta interesante conocer cómo fue su desarrollo comercial.

Una de las etapas más importantes en la configuración del Pisco fue entre 1882 y 1930 . En este periodo se establecieron las bases de la identidad del producto. No fue un tema lineal ni fácil; hubo mucha confusión durante un tiempo largo, con mensajes cruzados y contradicciones, en un contexto fascinante de batallas por la identidad del producto. Buena parte de esos conflictos se resolvieron el 15 de mayo de 1931, cuando el presidente Ibáñez delimitó la DO Pisco por el Decreto con Fuerza de Ley número 181.

Para conocer esta crítica etapa de la génesis del Pisco, resulta oportuno el análisis de las etiquetas. El

\footnotetext{
Estudios Internacionales (USACH), Santiago, Chile.

Escuela Nacional de Sommelier, Santiago, Chile.

Universidad de Santiago de Chile (USACH), Santiago, Chile.

Autor por correspondencia: pablo.lacoste@usach.cl
} 
estudio de los marbetes es un campo de conocimiento relativamente nuevo. En los últimos años, diversos autores han comenzado a cultivarlo. Una primera etapa es la reunión de etiquetas y carteles para crear corpus documentales que sirvan de base para la investigación. Varias obras, muchas de ellas de carácter institucional, se han dedicado a reunir y publicar etiquetas tanto en Europa como en América (VVAA, 1990; Frutos y Beretta, 1999; Crestin, 2000; VVAA, 2006; Álvarez Caselli, 2008; Inapi, 2010; VVAA, 2012; AGN 2015). El paso siguiente es el análisis específico de las etiquetas como fuente de información para conocer los elementos que hay detrás de ellas, proceso reflejado en una línea de investigación precisa y de fecundos resultados (Ramos Santana, 1995; Cirici, 1995; Tresaco, 2010; Miranda y Coutinho, 2010). Profundizando esa línea de trabajo, este artículo examina las etiquetas de Pisco en su primer medio siglo de historia.

Para la presente investigación se han considerado las etiquetas de Pisco conservadas en el Instituto Nacional de Propiedad Industrial (INAPI) de Chile, desde los primeros registros (1882) hasta las vísperas de la delimitación del Pisco como DO (1930). Dentro de este periodo, el INAPI conserva en sus archivos 111 etiquetas de Pisco chileno. Para establecer un marco comparativo, se han considerado también las etiquetas de Pisco peruano. En el caso de las registradas en Perú, se han considerado las recopilaciones realizadas por la historiografía peruana (VVAA, 1990 y AGN, 2015), correspondiente a 12 marbetes; a ello se suman otras tres registradas en Chile para su comercialización dentro de este país.

A primera vista podría parecer muy asimétrica la comparación de 111 etiquetas chilenas con 15 peruanas. De todos modos, esta situación parece reflejar el grado de desarrollo de ambas industrias en ese periodo histórico. Como ha señalado la literatura especializada peruana, el pisco de ese país se ha desarrollado con fuerza en los últimos cincuenta años. Previo a ello sufrió una serie de dificultades por varias causas: por un lado se arrancaron muchas viñas para dedicar las tierras a cultivos más rentables como el algodón y la caña de azúcar; por otro, la abundancia de las plantaciones de caña generó la abundancia de alcohol hecho de esta materia prima, cuyos costos eran inferiores en $50 \%$ al destilado de uva, esto promovió un ambiente de falsificación y adulteración: con frecuencia se vendían aguardientes de caña como si fuesen de uva, con el consiguiente impacto negativo en la industria pisquera peruana
(Rovira, 1966; Huertas, 2012; Onuki, 2014). En este contexto, es lógico que en el periodo indicado se hayan registrado más etiquetas chilenas que peruanas. De todos modos, esta investigación es falseable: futuras investigaciones en archivos de organismos del Perú o en el Indecopi, análogo de INAPI en Perú, podrían aportar nuevos datos relativos a etiquetas peruanas y ofrecer así nuevas conclusiones. Por el momento esta investigación examina las fuentes actualmente disponibles.

El análisis de las etiquetas se ha focalizado en tres puntos: 1. definición de la denominación del producto; 2 . tipo de uva; 3 . origen geográfico.

\section{Denominación del producto}

El primer punto saliente es la temprana consolidación de la palabra "pisco" para denominar al producto en Chile. En 1882 se registró la primera etiqueta con este nombre en el INAPI. A partir de allí, todos los marbetes se protocolizaron con este concepto. En total, las 111 etiquetas registradas de Pisco chileno entre 1882 y 1930 coincidieron en denominar "pisco" al aguardiente de uva.

La primera marca de pisco registrada formalmente fue Pisco G (1882) y Goyenechea (1886), elaborado en Copiapó (Figuras 1 y 2). En ambos casos se destacaba el producto como Pisco y reivindicaba el origen geográfico de la zona pisquera tradicional. La etiqueta de Pisco $G$ o Goyenechea incluía las siglas "M. de F.", es decir, "Marca de Fábrica". Como decoración, la etiqueta de 1882 presentaba una mesa con botellas negras, copas y racimos de uva. Cuatro años después, en 1886, la empresa avanzó en el diseño de una nueva etiqueta que, en lo fundamental, mantenía la identidad definida en la anterior.

El diseño de la segunda etiqueta de los Goyenechea, junto con mantener varios elementos del marbete anterior, aporta también una novedad interesante: el parrón. Se trata de una forma de cultivar la cepa que se caracteriza por el uso de horcones como sostén. Estos eran pilares de madera bastante altos, lo que requería mayores costos pero, a la vez, entregaba algunas ventajas significativas. En el período colonial los parrones eran parte de la vivienda, pues funcionaban como un salón al aire libre, una prolongación de los corredores. Allí se colocaban mesas y asientos para realizar diversas tareas domésticas durante el día y también para compartir los alimentos a la hora de comer. El parrón, 


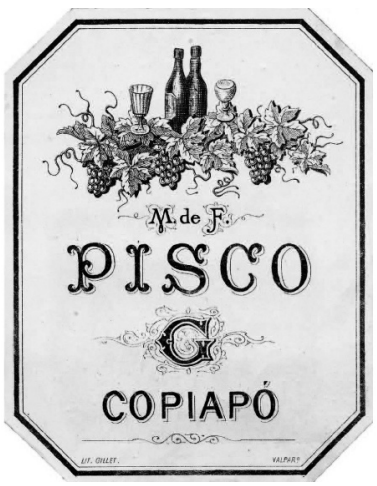

Figura 1. Etiqueta de "Pisco G".

Fuente: Archivo del Instituto Nacional de Propiedad Industrial de Santiago de Chile (en adelante INAPI), registro $N^{\circ} 304$ (10-2-1882).

en las casas de campo chilenas, era un espacio social de encuentro entre familiares y amigos. Pero en el caso del Norte Chico, los parrones necesitaron un desarrollo mayor para lograr efectos sobre la uva, particularmente para protegerla de los rayos directos del sol durante el verano. En cierta forma, los parrones fueron una característica importante en los paisajes vitícolas de Coquimbo; y esta etiqueta de los Goyenechea reivindicaba este lazo entre el Pisco y el paisaje de los parrones.

Junto con las anteriores, la tríada fundadora se completó con la etiqueta de Pisco Ytalia registrada en 1883 por el exgobernador del departamento de Elqui, don Juan de Dios Pérez de Arce (Figura 3). Igual que en las dos anteriores, este marbete reivindicaba el nombre pisco para llamar al producto y el origen geográfico de él, esta vez, en el valle del Elqui. Con notable alarde en economía de palabras, la etiqueta definía la naturaleza del producto (pisco), la variedad de la uva empleada (Moscatel de Alejandría), el lugar de origen (valle del Elqui) y el nombre del propietario (Pérez de Arce). El registro se formalizó en 1883 y la estiqueta fue publicada en el libro general de historia del diseño industrial chileno (INAPI, 2010: 46).

Las etiquetas de Pisco Goyenechea de Copiapó (1882/1886) y de la fábrica de pisco de don Juan de Dios Pérez de Arce en Vicuña (1883) establecieron las características de la identidad de los piscos del Norte Chico de Chile y marcaron la tendencia de lo que vendría después. Estos registros permitieron establecer las bases de un estilo que se fue consolidando con el tiempo, a medida que otros productores tomaban consciencia de la relevancia

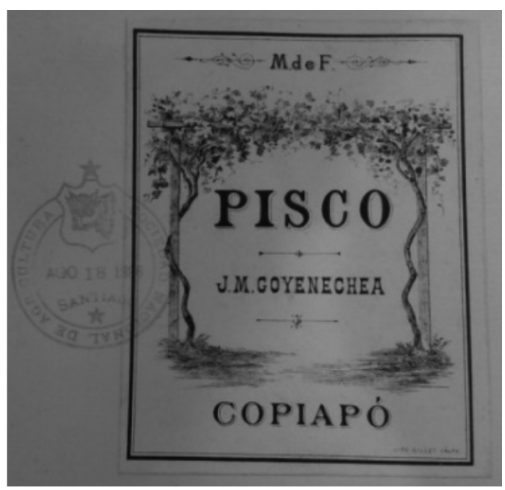

Figura 2. Etiqueta de "Pisco Goyenechea". Fuente: INAPI, registro No 125 (18-8-1886).

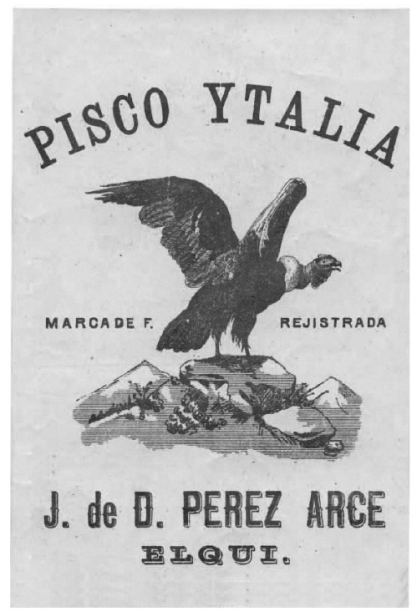

Figura 3. "Pisco Ytalia" de Juan de Dios Pérez de Arce. Fuente: INAPI, registro No 334 (13-3-1883).

de los trámites legales ante la oficina del Estado, como herramienta adecuada para fortalecer la identidad del producto. En los años siguientes, diferentes viticultores del Norte Chico siguieron este camino: entre 1894 y 1901 se registraron otros seis marbetes con el nombre "pisco" para denominar al producto chileno (Figuras 4, 5, 6, 7 y 8). En los años posteriores esta tendencia se consolidó. Para 1930 ya se habían registrado 111 etiquetas de "pisco" chileno utilizando este nombre (pisco) para denominar al producto.

Esta situación contrasta notablemente con el Perú. Los productores de este país usaron diversos conceptos para registrar sus productos: aguardiente, puro de Ica, entre otras expresiones, se usaron para 


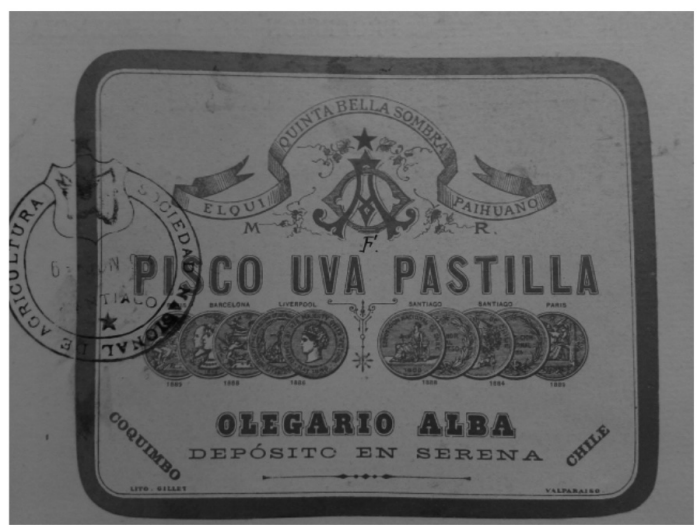

Figura 4. Pisco Olegario Alba.

Fuente: INAPI, registro No 494 (4-6-1894).

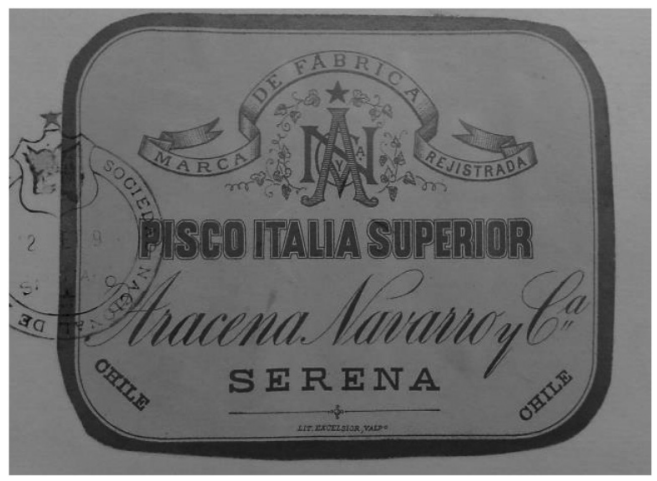

Figura 5. Pisco Aracena Navarro y Cía.

Fuente: INAPI, registro No 571 (16-9-1895).

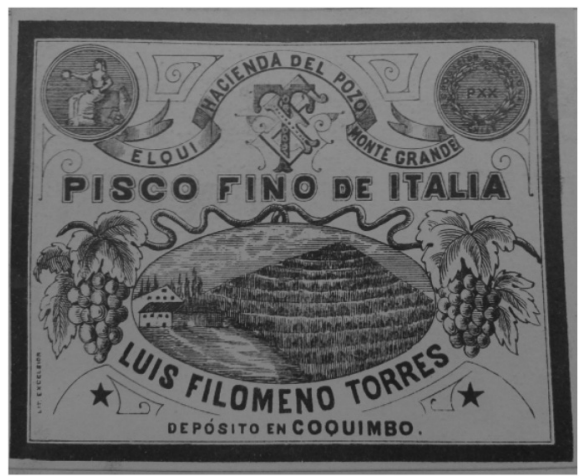

Figura 6. Pisco Luis Filomeno Torres.

Fuente: INAPI, registro $N^{\circ} 772$ (7-7-1897).

denominar al producto (Figura 9). De acuerdo con el actual estado de los conocimientos sobre el tema, el registro más antiguo realizado en Perú con la palabra "pisco" para llamar al producto data de 1922 (Figura 10). Esto implica 40 años después

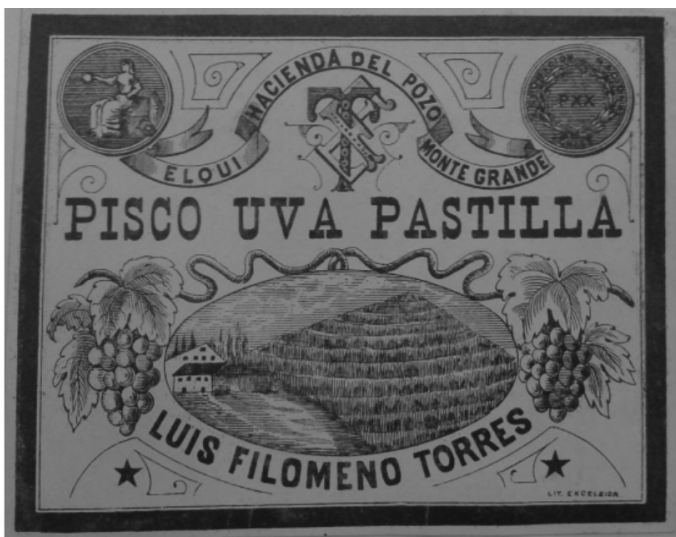

Figura 7. Pisco Luis Filomeno Torres.

Fuente: INAPI, registro $N^{\circ} 773$ (7-7-1897).

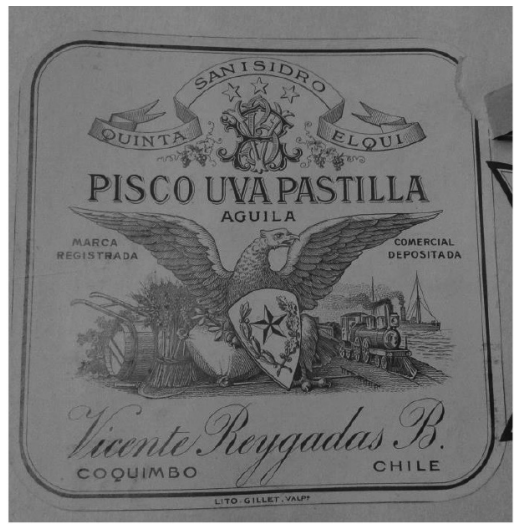

Figura 8: Pisco Águila.

Fuente: INAPI, registro No 1112 (23-3-1901).

que en Chile. La Tabla 1 entrega un perfil de las 15 etiquetas de destilados peruanos de aquel periodo.

La Tabla 1 muestra el complejo proceso que siguió la industria peruana para construir la identidad del producto. Hacia 1930, en Perú solo se habían registrado cinco etiquetas de pisco, mientras que en Chile, los productores nacionales tenían ya 111 marbetes de pisco. Como se ha señalado, en Perú recién se comenzó a usar el concepto pisco para denominar al producto en las etiquetas a partir de 1922. En cambio en Chile los productores nacionales ya habían resuelto este tema en 1882, es decir, 40 años antes. ¿Hubo algún tipo de influencia de Chile en Perú? ¿Cómo se comercializaron los aguardientes peruanos en Chile en esa época?

La tendencia inicial de los comerciantes peruanos en el mercado chileno era igual a la que desarrollaban en Perú. Los aguardientes se vendían con diversos nombres, sin usar, en la primera etapa, 
Tabla 1. Etiquetas registradas de Pisco peruano en Chile y Perú (1882-1930).

\begin{tabular}{lllll}
\hline Año & \multicolumn{1}{c}{ Marca } & \multicolumn{1}{c}{ Producto } & \multicolumn{1}{c}{ Empresario } & \multicolumn{1}{c}{ Lugar } \\
\hline 1886 & Cinto y Matog. & Puro & C \& M & Locumba \\
1890 & Ant. BodNac. & s/d & Pedro Coda & Tacna \\
1910 & & Pisco Superior & Denis Hnos. & Locumba \\
1919 & Ocucaje & Agte Puro & Zunini y Ocello & Ica \\
1922 & Vidal & Pisco Superior & S. Vidal & Arequipa \\
1924 & Porcella & Pisco Superior & A. Porcela & Arequipa /Ica \\
1924 & El Sol & Pisco Especial & Cía. Vinícola El Sol & S/d \\
1927 & A la flor de Santa Rosa & Licores & C. Biondo & Callao \\
1927 & s/d & Puro de Ica & Lanzone Hnos. & Ica \\
1927 & D= racimo uva & Puro de Ica & José Rotondo & Ica \\
1927 & Solari Hnos & Agte. Puro & Solari Hnos. & Miraflores \\
1928 & Urquiza & Pisco Superior & Ildefonso Urquiza & Arequipa \\
1928 & Inca & s/d & M. Picasso Hnos. & Ica Villa Vista Alegre \\
1929 & Zuñiga (D= León) & Pisco Especial & C. Zúñiga & Moquegua/ Arequipa \\
\hline
\end{tabular}

Fuente: Elaboración propia a partir de las etiquetas publicadas en VVAA (1990) y AGN (2015), más los tres marbetes detectados en los repositorios del INAPI.

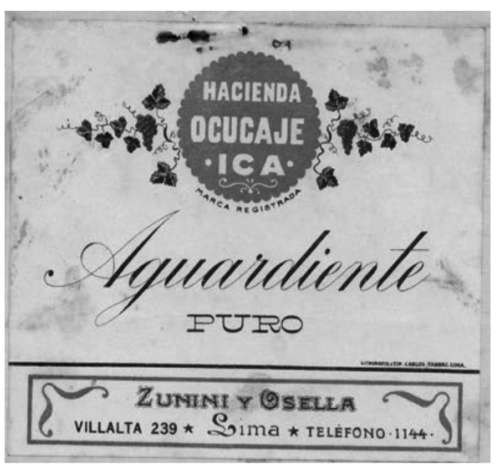

Figura 9. Etiqueta de aguardiente peruano registrado en Perú (1919). El producto se denomina "Aguardiente". Fuente: AGN (2015), p. 36 (documento 12).

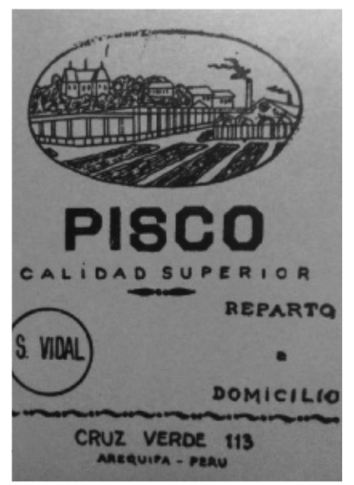

Figura 10. Primera etiqueta de pisco registrada en Perú (1922). Fuente: VVAA (1990), p. 92.

el concepto "pisco" para denominar al producto. Así se refleja en los registros de destilados peruanos en

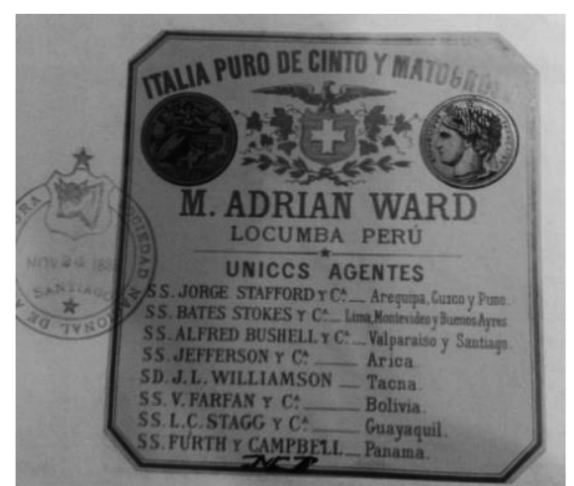

Figura 11. Primera etiqueta de aguardiente peruano registrada en Chile (1886). El producto se denomina "Italia Puro". No se usa la palabra "pisco".

Fuente: INAPI, registro No 137 (24-11-1886).

el INAPI chileno, realizados en 1886 (Figura 11) y 1890 (Figura 12). Se produjo entonces un proceso de desarrollos paralelos: cada producto recorría su camino con su propia identidad, sin cruzarse con el otro: los piscos chilenos se llamaban piscos, y los aguardientes peruanos se comercializaban como "aguardientes", "puro de Italia" y otros nombres. De todos modos, después de un tiempo, esto caminos terminarían por cruzarse.

La experiencia de los comerciantes peruanos dentro del mercado chileno generó una tendencia natural de adaptación al ambiente local para mejorar la sintonía cultural con los consumidores y mejorar las ventas. Esta tendencia se percibió en el diseño de las etiquetas. Los marbetes peruanos se 


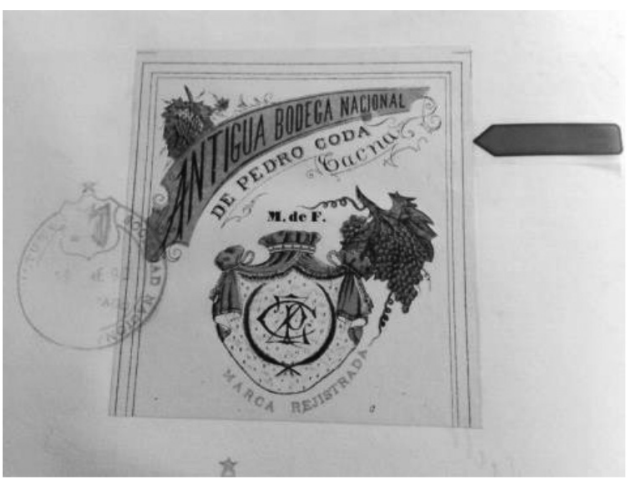

Figura 12. Segunda etiqueta de aguardiente peruano registrada en Chile (1890). No se usa la palabra pisco para denominar al aguardiente.

Fuente: INAPI, registro No 337 (14-1-1890).

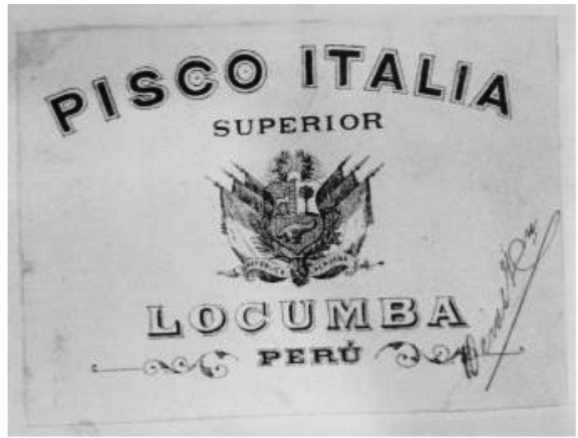

Figura 13. Etiqueta de pisco peruano registrado en Chile (1910). Es el marbete más antiguo de un destilado peruano que define el producto como "pisco".

Fuente: INAPI, registro No 6151 (9-8-1910).

fueron aproximando a las corrientes imperantes en Chile. Esta situación se hizo visible en tiempos del Centenario, cuando en los espacios públicos y las sensibilidades de época, se hizo común destacar los símbolos patrios, incluyendo escudos y banderas, para celebrar el primer siglo de la República. Dentro de esta tendencia peruana de adaptación al mercado chileno, surgió el marbete de 1910, el que exhibe el escudo patrio peruano, y por primera vez se emplea la palabra "pisco" (Figura 13). Esta etiqueta registrada en el INAPI representa el eslabón perdido entre la corriente principal de piscos chilenos en Chile (iniciada en 1882) y la línea de piscos peruanos en Perú (comenzada recién hacia 1922). La industria peruana asentada en Chile, en su esfuerzo por adaptarse a las condiciones del mercado local, adoptó el uso de este concepto para denominar al producto en 1910, y luego transfirió esta idea hacia el Perú, donde comenzó a usarla en 1922.

\section{Tipo de uva}

El tipo de uva también fue representado con intensidad en las etiquetas de pisco del periodo estudiado. Sobre el total de 111 marbetes, 78 mencionaban la variedad de uva con la que se elaboraba el producto: 53 de Moscatel Rosada (Uva Pastilla), 24 de Moscatel de Alejandría (Uva Italia) y una etiqueta mencionaba ambas variedades. Esto marca una tendencia importante: en la etapa de configuración del pisco como producto típico se reivindicaron las uvas tradicionales como materias primas de este aguardiente. Los productores sostuvieron que este destilado se apoyaba en variedades históricas. Una de ellas fue traída por los españoles a comienzos del siglo XVIII; la otra es una variedad criolla, surgida del cruce de la anterior con la otra variedad del periodo colonial, la Uva País.

El pisco original no tenía ningún contacto con las uvas francesas. Estas fueron incorporadas tardíamente a la categoría de uvas pisqueras, por decisión de la burocracia central. No fue parte genuina de la industria del pisco en su etapa fundacional.

\section{Origen geográfico}

El corpus documental generado por las etiquetas relevadas en el INAPI aporta elementos para conocer el proceso de construcción del prestigio del Pisco por medio de la visibilización de su lugar de producción. Del total de 111 etiquetas de Pisco chileno registradas en los 48 años examinados, la mención de lugar geográfico se presentó en 101 casos, esto representa el 90\% del total. Este elevado porcentaje demuestra que para el mercado del Pisco era particularmente importante el vínculo del producto con un determinado territorio. En ese sentido, es esencial destacar que ninguna otra información era tan recurrente en las etiquetas: ni los diseños, ni la referencia a la materia prima, ni la exaltación de las medallas obtenidas en las exposiciones internacionales.

Dentro del grupo de 101 etiquetas de Pisco con referencia a lugar geográfico, 85 corresponden a la zona histórica de producción, en el Norte Chico, mientras que las 16 restantes mencionaban otros lugares. Se produjeron verdaderas batallas comerciales y culturales en torno a la identidad del Pisco, aspecto de singular relevancia, ya que se trataba de un periodo fundacional dentro de la historia del producto. 
Los diseñadores adoptaron diversos criterios para transmitir el mensaje geográfico en las etiquetas de Pisco asociadas a la zona productiva histórica del Norte Chico. Algunos destacaban el nombre de la provincia en general (Coquimbo). Otros preferían acotar el territorio a un valle en particular (Copiapó, Huasco, Elqui, Limarí). También hubo casos más precisos, en los cuales se indicaba la localidad dentro del valle (Diaguitas, Paihuano, Rivadavia, La Unión). Resulta interesante ver estos detalles en su conjunto (Tabla 2).

Dentro de las etiquetas con identificación geográfica la mayor parte corresponde a la zona de producción histórica de Pisco en Chile; se trata de un amplio territorio ubicado al sur del desierto de Atacama, entre el valle de Copiapó por el norte y el valle de Limarí por el sur. Este territorio fue reivindicado por 85 etiquetas de Pisco. Esta información muestra el profundo arraigo histórico, cultural, económico y social que tenía el Pisco en esta zona histórica de producción. Y también coincide con el territorio delimitado por el DFL 181 de 1931.

La identificación geográfica de las etiquetas de Pisco muestra el profundo arraigo que las zonas de producción tenían como base de la calidad del producto. Eran el principal argumento de la construcción de la imagen del Pisco chileno. Por este

Tabla 2: Etiquetas de Pisco del Norte Chico de Chile registradas en el INAPI (1882-1930).

\begin{tabular}{|c|c|c|c|}
\hline \multicolumn{2}{|c|}{ Lugar geográfico } & \multicolumn{2}{|c|}{$\mathrm{N}^{\mathrm{o}}$} \\
\hline \multirow[t]{2}{*}{ Copiapó } & & & \\
\hline & Huasco & & \\
\hline \multirow[t]{2}{*}{ Huasco } & San Félix & & \\
\hline & Subtotal Huasco & & \\
\hline \multirow[t]{9}{*}{ Elqui } & Diaguitas & & \\
\hline & Paihuano & & \\
\hline & Tres Cruces & & \\
\hline & La Unión & & \\
\hline & Rivadavia & & \\
\hline & Monte Grande & & \\
\hline & La Serena & & \\
\hline & Elqui en general & & \\
\hline & Subtotal Elqui & & \\
\hline \multirow[t]{4}{*}{ Limarí } & Ovalle & & \\
\hline & Monto Dotris & Rapel & 5 \\
\hline & Mionte Patria & Tulahuén & 3 \\
\hline & Subtotal Limarí & & \\
\hline \multicolumn{2}{|c|}{ Subtotal valles internos definidos } & & \\
\hline \multicolumn{2}{|c|}{ Coquimbo en general } & & \\
\hline \multicolumn{2}{|c|}{ Total zona pisquera } & & \\
\hline
\end{tabular}

motivo, los productores se esmeraron en lucir los nombres de las zonas productivas en las etiquetas.

En total se registraron 16 etiquetas con indicación geográfica fuera de la zona tradicional de producción de Pisco. Estos se ubicaban preferentemente en las grandes ciudades chilenas, sobre todo Santiago (6), Valparaíso (3) y Concepción (3). En menor medida se registraron etiquetas de zonas rurales del Valle Central (Rancagua: 2; Itata: 1) o del sur (Puerto Montt: 1). Los que se interesaban en lucrar con la fama del pisco no eran los productores artesanales de la zona central de Chile, sino los comerciantes asentados en las grandes ciudades.

La batalla por la identidad del concepto pisco, dentro de Chile, se libró entre los productores del Norte de Chico, por un lado, y los grandes comerciantes de Santiago, Valparaíso y Concepción, por otro. Por tanto, era una lucha de poder entre los campesinos, que vivían en el interior de los valles transversales del Norte Chico, cultivando sus viñas, contra las empresas comerciales asentadas en las grandes ciudades, que requerían un producto famoso para incrementar sus ventas. En esta lucha desigual, los tecnócratas y agrónomos, como Manuel Rojas (1891, 1897), estuvieron del lado de la deslocalización del Pisco, e indirectamente, apoyaron el partido de los grandes comerciantes en detrimento de los productores de las zonas pisqueras.

Los productores del Norte Chico, mediante sus representantes en el Parlamento chileno, lograron hacer oír su voz y hacer sentir sus aspiraciones ante el Estado Nacional. El tema fue debatido al más alto nivel. Durante largos años el gobierno de Chile reunió los antecedentes para buscar una solución al problema. Fue un conflicto territorial dentro de Chile, un capítulo más dentro de la "Guerra del Pisco". Estas tensiones se reflejaron en los considerandos del DFL 181, del 15 de mayo de 1931. En este documento se señala que:

Desde antiguo se elaboran aguardientes en las zonas de Huasco y Elqui, que con el nombre de pisco han alcanzado justo renombre en el país y en el extranjero, gracias a las condiciones especiales de la región y de la calidad de las uvas cuyos caldos se destilan;

Sin embargo, la fama construida por los productores del Norte Chico atrajo el interés de otros sectores, que intentaron lucrar con el esfuerzo 
ajeno. Así lo señalaba el mismo DFL 181 en sus considerandos:

La competencia que le hacen otros productos similares fabricados en otras zonas ha restringido el mercado conquistado a fuerza de tantos años y sacrificios por la venta de substitutos que por su naturaleza pueden expenderse a más bajo precio (...) Este estado de cosas ha traído la consiguiente desvalorización de la industria pisquera, impulsándola desde poco tiempo a esta parte a una aguda crisis.

A ello se añaden otros elementos interesantes:

El pisco propiamente tal, que proviene de la destilación de los caldos de uva, se produce sólo en las zonas comprendidas entre Huasco y el río Limarí. (...) Los que con el mismo nombre se elaboran más al Sur, son extraídos de orujos y otras materias primas que no son de uva, en cantidades que a veces duplica la legítima producción que corresponde a las zonas nombradas.

La argumentación del DFL 181 reunía y sintetizaba los fundamentos agrupados por el Ministerio de Agricultura. El argumento central era el prestigio y la fama que habían logrado los productores del Norte Chico, gracias al esfuerzo por elaborar productos de mayor calidad, a lo largo de los años. Las Exposiciones Universales y los registros de marcas contribuyeron a fortalecer esta fama, ello facilitó la compilación de antecedentes suficientes para fundamentar la Delimitación del pisco como Denominación de Origen. Este fue el sentido del DFL 181, firmado el 15 de mayo de 1931. A partir de entonces solo se pudo utilizar el nombre de Pisco para llamar los aguardientes elaborados en los valles del Norte Chico de Chile. De esta manera quedó delimitada la primera DO de América.

\section{Conclusión}

La colección de etiquetas registradas en los archivos del INAPI constituye una fuente de singular valor para conocer la evolución de los productos agroalimentarios, como revela el caso del Pisco. Por los marbetes se ha podido conocer su temprana definición como denominación (pisco), lugar geográfico (Norte Chico de Chile) y variedades de uvas (Moscatel de Alejandría y Moscatel Rosada).

Se muestra también la primacía del Pisco chileno sobre el peruano: en Chile se comenzó a llamar "pisco" al producto en 1882; en cambio en Perú esta denominación se comenzó a utilizar 40 años más tarde, a partir de 1922. Entre ambos puntos se ha detectado un eslabón perdido, que es una etiqueta peruana registrada en Chile en 1910; al parecer, la industria peruana aprendió el uso del nombre pisco en Chile, y luego lo trasladó a su país. La brecha de tiempo que surgió a partir de las etiquetas era un reflejo del grado de madurez de la industria en ambos países. Y esta asimetría se reflejó en los procesos institucionales: el Estado de Chile delimitó la DO Pisco por ley en 1931, mientras que en Perú la ley respectiva se sancionó recién en 1994. Las etiquetas ya estaban preanunciando lo que ocurriría a nivel legal.

Desde el punto de vista del lugar geográfico de origen, el Pisco se elaboraba en el Norte Chico principalmente; también intentaron usar este nombre los grandes comerciantes de Santiago, Valparaíso y Concepción, para mejorar sus ventas. Pero este problema fue resuelto por el DFL 181 de 1931 que delimitó la DO Pisco en Chile.

Respecto del tema de las variedades de uva: la fuente muestra que las cepas originales del Pisco chileno eran la Moscatel de Alejandría, variedad histórica, introducida por los españoles a comienzos del siglo XVIII, y la Moscatel Rosada, vidueño criollo, descendiente de la cruza de la anterior con la Uva País. No había variedades de uva francesa. A diferencia de los temas anteriores, la identidad vitícola del Pisco no fue definida por el DFL 181; tampoco se protegió esta tradición por un Consejo Regulador, que nunca se creó. El tema quedó desprotegido, y expuesto a cambios y distorsiones que ocurrieron después, en los años 60 y 70, cuando los tecnócratas del SAG, arbitrariamente, incorporaron otras variedades exóticas, debilitando la identidad de las uvas pisqueras.

\section{Agradecimientos}

Los autores agradecen al Proyecto Fondecyt 1130096. 


\section{Literatura Citada}

Aillón, E.; Kirigin, M.A.

2013. San Pedro: testigo de los tiempos. Por la ruta del singani en Bolivia. Siglos XVI-XXI. La Paz. Archivo y Biblioteca Nacional de Bolivia, 798 p.

Álvarez Caselli, P.

2008. Chile marca registrada. Historia general de las marcas comerciales y el imaginario del consumo en Chile. Santiago. Universidad del Pacífico, 343 p.

Arancibia, M.J.

2015. La importancia de las Denominaciones de Origen e Indicaciones Geográficas para la identidad país en Chile. En: I Seminario Productos Típicos, Denominaciones de Origen y Desarrollo Rural. Santiago. USACH. 10 y 11 de setiembre de 2015, pp. 85-98.

Archivo General de la Nación (AGN).

2015. Entre viñedos y botijas. Producción, consumo y comercio del Pisco, 1568-2013. Lima. Archivo General de la Nación, $112 \mathrm{p}$.

Belmar, C.

2015. Las Denominaciones de Origen en Chile: Desarrollo y Perspectivas. En: I Seminario Productos Típicos, Denominaciones de Origen y Desarrollo Rural, Santiago, USACH, 10 y 11 de setiembre de 2015, pp. 75-84.

Buitrago, A.S.

2015. La vitivinicultura emergente en Bolivia y las oportunidades para el Singani. RIVAR, 1 (2): 87-101.

Castro, A.; Mujica, F.; Argandoña, F.

2015. Entre Pintatani y Codpa. Paisaje y productos típicos en los relatos campesinos, 1847-2013. RIVAR, 2 (6): 70-86.

Chaves, N.

2008. La marca de la historia, pp. 14-15. En: Álvarez Caselli, Pedro. 2008. Chile marca registrada. Historia general de las marcas comerciales y el imaginario del consumo en Chile. Santiago. Universidad del Pacífico, 343 p.

Crestin-Billet, F.

2000. La folie des etiquettes de vins. París. Flammarion, 2003, 384 p.

Cirici Narváez, J.R.

1995. La estética de las etiquetas antiguas del Vino Fino. En: Actas de las I Jornadas del Vino Fino. Historia, Arte y Mentalidades. Puerto de Santa María. Ayuntamiento de Santa María, pp. 79-98.

Dargent Chamot, E.

2013. Vino y pisco en la historia del Perú. Lima. Fondo Editorial Universidad San Martín de Porres, 165 p.

Frutos, E., Beretta, A.

1999. Un siglo de tradición. Primera historia de uvas y vinos del Uruguay. Montevideo. Aguilar, 242 p.

Gutiérrez, G.

2005. El pisco. Apuntes para la Defensa Internacional de la Denominación de Origen Peruana. Lima. Fondo Editorial del Congreso del Perú, 209 p.

Hernández López, J.J.

2013. Paisaje y creación de valor. La transformación de los paisajes culturales del agave y del tequila. Zamora, Michoacán. El Colegio de Michoacán/Fideicomiso "Felipe Teixidor y Monserrat Alfau de Teixidor", 349 p.

Huertas, L.

2012. Cronología de la producción del vino y del Pisco (Perú 1548-2010). Lima. Editorial Universitaria, 393 p.
Instituto Nacional de Propiedad Industrial (INAPI).

2010. Historia gráfica de la propiedad industrial en Chile. Santiago de Chile, 193 p.

Jiménez Cabrera, D.

2014. La consolidación de la Denominación de Origen Pisco (1931): una mirada desde los debates parlamentarios y sus referentes internacionales (1901-1931). RIVAR, 1 (2): 72-89.

Lacoste, P.; Jiménez, D.; Castro, A.; Rendón, B.; Soto, N.

2013. A binational Appellation of Origin: Pisco in Chile and Peru. Chilean journal of agricultural research, 73 (4): 424-429.

Lacoste, P; Navarrete, S.

2014. Alternativas no tradicionales de desarrollo rural: la ruta del pisco como recurso turístico (Valle de Elqui, Chile). Idesia, 32 (4): 5-14.

Lacoste, P.; Jiménez, D.; Cruz, E.; Rendón, B.; Soto, N.; Solar, M.; Polanco, C.

2014. Rutas del aguardiente en el Cono Sur de América (siglos XVI-XIX) Antecedentes de la Denominación de Origen Pisco. Idesia, 32 (3): 43-50.

Lacoste, P.; Jiménez, D.; Cruz, E.; Rendón, B.; Soto, N.

2014. Pisco y toponimia: impacto de las rutas del aguardiente en el desarrollo de nombres y lugares geográficos en Chile, Perú y Argentina. Idesia, 32 (3): 31-41.

Lacoste, P.; Briones, F.; Jiménez, D. y Rendón, B.

2014. La Denominación de Origen Pisco en Chile: algunos problemas nacionales e internacionales. Idesia, 32 (2): 47-56.

López Barrera, M.D.

2015. Las denominaciones de origen en México y sus implicancias culturales. En: I Seminario Productos Típicos, Denominaciones de Origen y Desarrollo Rural. Santiago. USACH, 10 y 11 de setiembre de 2015, pp. 51-54.

Miranda, F.; Coutinho, M.A.

2010. Las etiquetas como género de texto: un abordaje comparativo. En: Ibáñez Rodríguez, Miguel. Vino, lengua y traducción. Universidad de Valladolid, Volumen 2 (CDRom): 315-334.

Onuki, Y.

2014. Dos rones: un estudio acerca de la difusión del cañazo en la sierra del Perú. Perspectivas Latinoamericanas, 11: 127-143.

Ramos Santana, A.

1995. Consideraciones en torno a las mentalidades en etiquetas de Vino Fino. En: Actas de las I Jornadas del Vino Fino. Historia, Arte y Mentalidades. El Puerto de Santa María, Ayuntamiento de El Puerto, pp. 61-78.

Rojas, M.

1891. Tratado de viticultura y vinificación. $1^{\circ}$ edición. Imprenta de la Libertad. Talca, Chile, 667 p.

Rovira, F.

1966. La industria del pisco en el Perú. Lima. Instituto Nacional de Promoción Industrial / Banco Industrial del Perú. 56 p.

Souza Borda, A.L.

2015. Denominaciones de origen en Brasil: situación actual. Su desarrollo: jurisprudencia y avances de los productores. RIVAR, 2 (5): 1-21.

Tresaco, M.P.

2010. La etiqueta de las botellas de vino. Bodegas del siglo XXI del Somontano. En: Ibáñez Rodríguez, Miguel. Vino, lengua y traducción. Universidad de Valladolid, Volumen 2 cdrOM: 291-314. 
VVAA.

1990. Crónicas y relaciones que se refieren al origen y virtudes del Pisco, bebida tradicional y patrimonio del Perú. Lima. Banco Latino, 135 p.

VVAA.

2006. Un siglo de carteles en la agricultura española 18701960. Colección de Carlos Velasco Murviedro. Madrid,
Ministerio de Agricultura, Pesca y Alimentación / Sociedad Anónima Estatal de Caución Agraria, 166 p.

VVAA.

2012. Bebidas espirituosas en España. Un recorrido por la historia del sector. Carteles 1873-1969. Colección de Carlos Velasco Murviedro. Madrid. Gobierno de España / Federación Española de Bebidas Espirituosas, 159 p. 